\title{
HIDDEN SYMMETRIES VIA HIDDEN EXTENSIONS
}

\author{
ERIC CHESEBRO AND JASON DEBLOIS
}

\begin{abstract}
This paper introduces a new approach to finding knots and links with hidden symmetries using "hidden extensions", a class of hidden symmetries defined here. We exhibit a family of tangle complements in the ball whose boundaries have symmetries with hidden extensions, then we further extend these to hidden symmetries of some hyperbolic link complements.
\end{abstract}

A hidden symmetry of a manifold $M$ is a homeomorphism of finite-degree covers of $M$ that does not descend to an automorphism of $M$. By deep work of Margulis, hidden symmetries characterize the arithmetic manifolds among all locally symmetric ones: a locally symmetric manifold is arithmetic if and only if it has infinitely many "non-equivalent" hidden symmetries (see [13, Ch. 6]; cf. [9]).

Among hyperbolic knot complements in $S^{3}$ only that of the figure-eight is arithmetic [10], and the only other knot complements known to possess hidden symmetries are the two "dodecahedral knots" constructed by Aitchison-Rubinstein [1]. Whether there exist others has been an open question for over two decades [9, Question 1]. Its answer has important consequences for commensurability classes of knot complements, see [11] and [2].

The partial answers that we know are all negative. Aside from the figure-eight, there are no knots with hidden symmetries with at most fifteen crossings [6] and no two-bridge knots with hidden symmetries [11]. Macasieb-Mattman showed that no hyperbolic $(-2,3, n)$ pretzel knot, $n \in \mathbb{Z}$, has hidden symmetries [8]. Hoffman showed the dodecahedral knots are commensurable with no others [7].

Here we offer some positive results with potential relevance to this question. Our first main result exhibits hidden symmetries with the following curious feature.

Definition 0.1. For a manifold $M$ (possibly with boundary) and a submanifold $S$ of $M$, a hidden extension of a self-homeomorphism $\phi$ of $S$ is a hidden symmetry $\Phi: M_{1} \rightarrow M_{2}$ of $M$, where $p_{i}: M_{i} \rightarrow M$ are connected, finite-sheeted covers for $i=1,2$, that lifts $\phi$ on a component of $p_{1}^{-1}(S)$.

We use a family $\left\{L_{n}\right\}$ of two-component links constructed in previous work [3]. For each $n, L_{n}$ is assembled from a tangle $S$ in $B^{3}, n$ copies of a tangle $T$ in $S^{2} \times I$, and the mirror image $\bar{S}$ of $S$. Figure 1 depicts $L_{2}$, with light gray lines indicating the spheres that divide it into copies of $S$ and $T$. For $n \in \mathbb{N}$ and $m \geq 0$, we will also use a tangle $T_{n} \subset L_{m+n}$ : the connected union of $S$ with $n$ copies of $T$. For instance, $L_{2}$ contains a copy of $T_{1}$ (which is pictured in Figure 2 below) and of $T_{2}$.

Upon numbering the endpoints of $T_{n}$ as indicated in Figure 2, order-two even permutations determine mutations: mapping classes of $\partial\left(B^{3}-T_{n}\right)$ induced by 180-degree rotations of the sphere obtained by filling the punctures.

Theorem 1.8. For $n \in \mathbb{N}$, the mutation of $\partial\left(B^{3}-T_{n}\right)$ determined by (13)(24) has a hidden extension over a cover of $B^{3}-T_{n}$ and for any $m \in \mathbb{N}$, taking $T_{n} \subset L_{m+n}$, a hidden extension over a cover of $S^{3}-L_{m+n}$. 


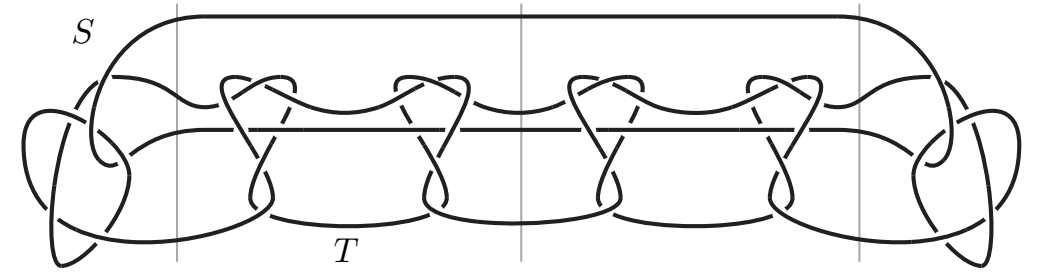

Figure 1. The link $L_{2}$

In particular, this gives the first proof that the $S^{3}-L_{m+n}$ have hidden symmetries. Its heart is the fact that though $(13)(24)$ does not extend over $S^{3}-L_{m+n}$, it is represented by an isometry of the totally geodesic $\partial\left(B^{3}-T_{n}\right)$ that is induced by an isometry $\mathrm{m}_{1}^{(n)}$ of $\mathbb{H}^{3}$ in the commensurator (see eg. [9, p. 274]) of the group $\Gamma_{m+n}$ uniformizing $S^{3}-L_{m+n}$. Lemma 1.6 asserts the analogous fact for the group $\Delta_{n}$ uniformizing $B^{3}-T_{n}$, which implies the other assertion of Theorem 1.8.

In Section 2 we attack the same problem on the same examples, but from a different direction. The idea in this section is to produce hidden symmetries without prior knowledge of an orbifold cover such as was used in Theorem 1.8. Instead we leverage the decomposition of $L_{n}$ into tangle complements, producing explicit hidden extensions of the mutation over covers of these and solving a gluing problem to piece them together to produce a hidden symmetry of $L_{n}$. One nice byproduct of this approach is an explicit description of the hidden symmetry. We show:

Theorem 2.9. For each $n \in \mathbb{N}$ there is an 11-sheeted cover $N_{n} \rightarrow B^{3}-T_{n}$ and a hidden extension $\Psi: N_{n} \rightarrow N_{n}$ of the mutation (13)(24) acting on $S^{(n)}-T_{n}$. Moreover, for each $m \in \mathbb{N}, \Psi$ extends to a hidden symmetry of an 11-sheeted cover of $S^{3}-L_{m+n}$ that contains $N_{n}$.

Given that we are motivated by hidden symmetries of knot complements, the following question is natural:

Question. Is there a knot $K$ in $S^{3}$ and a hidden symmetry of $S^{3}-K$ that is a hidden extension of a symmetry of some surface in $S^{3}-K$ ?

In fact as the referee has pointed out, one might ask this about the known examples with hidden symmetries. While it seems unlikely that the figure-eight knot complement has hidden extensions, given the classification of incompressible surfaces there (see $[12, \S 4.10]$ ), we have no corresponding conjecture about the dodecahedral knot complements. This would be interesting to know.

Another tantalizing possibility arises from the observation that each tangle $T_{n}$ also lies in many knots in $S^{3}$ which are distinct from the 3 known examples of knots with hidden symetries. If an analog of Theorem 2.9 could be proved for any such knot it would give a new example whose complement has hidden symmetries. We have ruled out many possibilities using a criterion given in [11, Corollary 2.2]: a knot complement with hidden symmetries has cusp field $\mathbb{Q}(i)$ or $\mathbb{Q}(\sqrt{-3})$. This condition can be easily checked with with SnapPy [5] and Snap (see [4]). We suspect that there is a reason the $T_{n}$ cannot lie in knots whose complements have hidden symmetries, and intend to study this further.

We conclude the introduction with two related problems. 


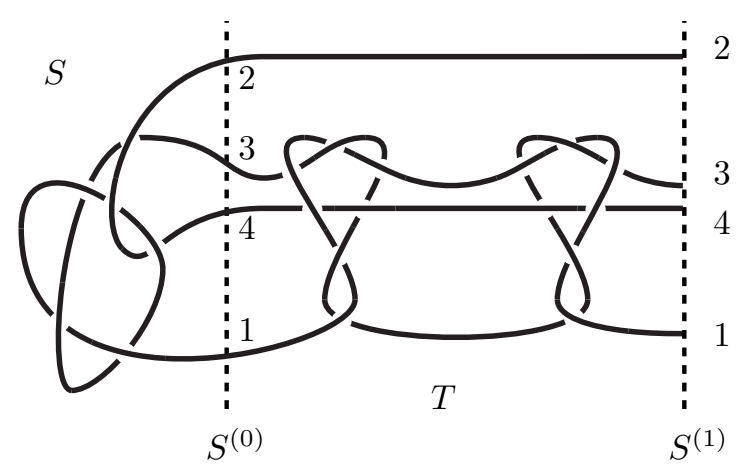

Figure 2. The tangle $T_{1} \subset B^{3}$ can be decomposed along a sphere $S^{(0)}$ into a tangle $S \subset B^{3}$ and a tangle $T \subset S^{2} \times I$.

Problem 1. Classify tangles in the ball with complements whose boundary has a symmetry with hidden extension.

Problem 2. Given a tangle $T$ in the ball $B^{3}$, and a symmetry $\phi$ of $\partial B^{3}-T$ with a hidden extension across a cover of $B^{3}-T$, classify the links $L$ containing $T$ such that the hidden extension of $\phi$ extends to a cover of $S^{3}-L$.

\section{EXISTENCE OF HIDDEN EXTENSIONS}

The goal of Section 1.1 is to describe the tangle complements $B^{3}-T_{n}$ from both the topological and geometric perspectives, by collecting relevant definitions and results scattered throughout [3] and re-assembling them here in a more helpful order. In this sub-section we merely summarize geometric details, referring the interested reader to [3] for proofs. In Section 1.2 we prove Theorem 1.8.

1.1. The topology and geometry of $T_{n}$. The solid lines in Figure 2 describe a two string tangle $T_{1} \subset B^{3} . \partial B^{3}$ is shown as a dotted line labeled $S^{(1)}$. There is an additional sphere $S^{(0)}$ shown in the figure. If we cut $\left(B^{3}, T_{1}\right)$ along $S^{(0)}$ we obtain a pair of tangles $\left(B^{3}, S\right)$ and $\left(S^{2} \times I, T\right)$. Orienting $I$ so that $S^{(0)}=S^{2} \times\{0\}$, we let $\partial_{-} T=T \cap S^{(0)}$ and $\partial_{+} T=T \cap S^{(1)}$.

Let $r_{T}:\left(S^{2} \times I, T\right) \rightarrow\left(S^{2} \times I, T\right)$ be the reflection homeomorphism visible in Figure 1, and let $T_{0}$ be the subtangle of $T$ that lies to the left of the fixed point set of $r_{T}$. That is, $T_{0}=T \cap S^{2} \times[0,1 / 2]$. Reparametrizing the underlying interval, we may also regard $T_{0}$ as a tangle in $S^{2} \times I$.

Proposition 1.1 below, which combines parts of Propositions 2.7, 2.8, and 3.7 of [3], introduces geometric models for the complements of the tangles $S, T_{0}$ and $T$. There and henceforth, we work with the upper half space model for $\mathbb{H}^{3}$ and use the standard representation of $\operatorname{Isom}\left(\mathbb{H}^{3}\right)$ as a $\mathbb{Z}_{2}$ extension of $\mathrm{PSL}_{2}(\mathbb{C})$. If $d \in \mathrm{PSL}_{2}(\mathbb{C})$, we write $\bar{d}$ for the matrix whose entries are the complex conjugates of the entries of $d$. When we apply this operation to each element of a subgroup $\Gamma<\mathrm{PSL}_{2}(\mathbb{C})$ we obtain a subgroup denoted by $\bar{\Gamma}$.

For a Kleinian group $\Gamma$, we denote the convex core of $\mathbb{H}^{3} / \Gamma$ as $C(\Gamma)$. We will use the term natural map as in [3] (see below Definition 3.1 there) to refer to the restriction to $C(\Lambda)$ of the orbifold covering map $\mathbb{H}^{3} / \Lambda \rightarrow \mathbb{H}^{3} / \Gamma$, for $\Lambda<\Gamma$. Because the limit set of $\Gamma$ contains that of $\Lambda$, the natural map takes $C(\Lambda)$ into $C(\Gamma)$. 
The geometric models for $B^{3}-S$ and $\left(S^{2} \times I\right)-T_{0}$ described in parts (1) and (2) of Proposition 1.1 are hyperbolic 3 -manifolds with totally geodesic boundary produced by pairing certain faces of the right-angled ideal octahedron and cuboctahedron, respectively, but they are described in the Proposition as convex cores of the quotients of $\mathbb{H}^{3}$ by the groups generated by the face-pairing isometries. The equivalence of these two forms of description is proved in Lemma 2.1 of [3].

Proposition 1.1. (1) For $\mathrm{s}=\left(\begin{array}{cc}1 & 0 \\ -1 & 1\end{array}\right)$ and $\mathrm{t}=\left(\begin{array}{cc}2 i & 2-i \\ i & 1-i\end{array}\right), \Delta_{0}=\langle\mathrm{s}, \mathrm{t}\rangle$ is $a$ Kleinian group, and there is a homeomorphism $f_{S}: M_{S} \doteq B^{3}-S \rightarrow C\left(\Delta_{0}\right)$.

(2) For $\mathrm{f}, \mathrm{g}$ and $\mathrm{h}$ below, $\Gamma_{T_{0}}=\langle\mathrm{f}, \mathrm{g}, \mathrm{h}\rangle$ is a Kleinian group, and there is a homeomorphism $f_{T_{0}}: M_{T_{0}} \doteq\left(S^{2} \times I\right)-T_{0} \rightarrow C\left(\Gamma_{T_{0}}\right)$.

$$
\mathrm{f}=\left(\begin{array}{cc}
1 & 0 \\
-1 & 1
\end{array}\right) \quad \mathrm{g}=\left(\begin{array}{cc}
-1+i \sqrt{2} & 1-2 i \sqrt{2} \\
-2 & 3-i \sqrt{2}
\end{array}\right) \quad \mathrm{h}=\left(\begin{array}{cc}
2 i \sqrt{2} & -3-i \sqrt{2} \\
-3+i \sqrt{2} & -3 i \sqrt{2}
\end{array}\right)
$$

(3) For $\mathrm{c}=\left(\begin{array}{cc}1 & i \sqrt{2} \\ 0 & 1\end{array}\right), \Gamma_{T}=\left\langle\Gamma_{T_{0}}, \mathrm{c}^{-2} \bar{\Gamma}_{T_{0}} \mathrm{c}^{2}\right\rangle$ is a Kleinian group, and there is a homeomorphism $f_{T}: M_{T} \doteq\left(S^{2} \times I\right)-T \rightarrow C\left(\Gamma_{T}\right)$ satisfying:

- composing the inclusion $M_{T_{0}} \rightarrow M_{T}$ with $f_{T}$ yields $f_{T_{0}}$; and

- for $r_{T}$ as above, $f_{T} \circ r_{T} \circ f_{T}^{-1}$ is induced by $x \mapsto \mathrm{c}^{-2} \bar{x} \mathrm{c}^{2}$.

(4) The intersection $\Delta_{0} \cap \Gamma_{T}$ is a Fuchsian group $\Lambda$ stabilizing the hyperplane $\mathcal{H}=\mathbb{R} \times(0, \infty)$ of $\mathbb{H}^{3}$. This is the intersection of the convex hulls of the limit sets of $\Delta_{0}$ and $\Gamma_{T}$, and the natural maps from $\mathcal{H} / \Lambda$ to $C\left(\Delta_{0}\right)$ and $C\left(\Gamma_{T}\right)$ map to totally geodesic boundary components.

(5) The image of the natural map $\mathcal{H} / \Lambda \rightarrow C\left(\Gamma_{T}\right)$ is the image of $\partial_{-} M_{T} \doteq$ $\left(S^{2} \times\{0\}\right)-T$ under $f_{T}$. The same holds with each instance of $T$ here replaced by $T_{0}$.

For the homeomorphism $j:\left(\partial B^{3}, \partial S\right) \rightarrow\left(S^{2} \times\{0\}, \partial_{-} T\right)$ such that $\left(B^{3}, S\right) \cup_{j}\left(S^{2} \times I, T\right) \cong\left(B^{3}, T_{1}\right), f_{T} \circ j \circ f_{S}^{-1}: \partial C\left(\Delta_{0}\right) \rightarrow C\left(\Gamma_{T}\right)$ factors through $\mathcal{H} / \Lambda$ as the composition of a natural map with the inverse of another.

We now turn back to topology and give an inductive definition of the tangles $T_{n}$, assembling $\left(B^{3}, T_{n}\right)$ from a single copy of $\left(B^{3}, S\right)$ and $n$ of $\left(S^{2} \times I, T\right)$ for each $n \in \mathbb{N}$, using $T_{1}$ as pictured in Figure 2 as the base case. Numbering the points of $\left(S^{(0)}, \partial T_{1}\right)$ and $\left(S^{(1)}, \partial T_{1}\right)$ as shown in the figure, let $\left(S^{2} \times\{1\}, \partial_{+} T\right)$, $\left(S^{2} \times\{0\}, \partial_{-} T\right)$, and $\left(\partial B^{3}, \partial S\right)$ inherit numberings from their inclusions to these spheres. Note that the resulting numbering of $\left(S^{2} \times \partial I, \partial T\right)$ is $r_{T^{-}}$-invariant.

Now for $n>1$, assume for $1 \leq k<n$ that tangles $T_{k} \subset B^{3}$ with labeled endpoints are defined, and, for $k>1$, inclusions $\left(B^{3}, T_{k-1}\right) \hookrightarrow\left(B^{3}, T_{k}\right)$ and $\iota_{k}:\left(S^{2} \times I, T\right) \rightarrow$ $\left(B^{3}, T_{k}\right)$, such that:

- $\left(B^{3}, T_{k}\right)=\left(B^{3} \cup \iota_{k}\left(S^{2} \times I\right), T_{k-1} \cup \iota_{k}(T)\right)$;

- $\iota_{k}$ preserves labels on $\partial_{+} T$; and

- the included image of $B^{3}$ intersects $i_{k}\left(S^{2} \times I\right)$ in a sphere $S^{(k)}$, with $\left(S^{(k)}, S^{(k)} \cap T_{k}\right)=\left(\partial B^{3}, \partial T_{k-1}\right)=\iota_{k}\left(S^{2} \times\{1\}, \partial_{+} T\right)$.

Define $T_{n} \subset B^{3}$ as the quotient of the disjoint union $\left(B^{3}, T_{n}\right) \sqcup\left(S^{2} \times I, T\right)$ by identifying $\iota_{n-1}(x, 1)$ to $(x, 0)$ for each $x \in S^{2}$; let the inclusion of $\left(B^{3}, T_{n-1}\right)$ and $\iota_{n}:\left(S^{2} \times I, T\right) \rightarrow\left(B^{3}, T_{n}\right)$ be induced by the respective inclusions into the disjoint union; and label the endpoints of $T_{n}$ coherently with $T \cap\left(S^{2} \times\{1\}\right)$ using $\iota_{n}$. It is clear by construction that the inductive hypothesis applies to $\left(B^{3}, T_{n}\right)$. 
Having topologically described the $T_{n}$, our next order of business is to give geometric models for their complements; that is to describe hyperbolic manifolds with totally geodesic boundary homeomorphic to the $B^{3}-T_{n}$. In parallel with our topological description of $T_{n}$, these are assembled from copies of the geometric models described in Proposition 1.1. To this end, we define:

$$
\Gamma_{T}^{(j)}=\mathrm{c}^{-2(j-1)} \Gamma_{T} \mathrm{c}^{2(j-1)} \quad \Lambda^{(j)}=\mathrm{c}^{-2 j} \Lambda \mathrm{c}^{2 j} \quad F^{(j)}=\mathrm{c}^{-2 j}(\mathcal{H}) / \Lambda^{(j)}
$$

Note for each $j$ that $C\left(\Gamma_{T}^{(j)}\right)$ is isometric to $C\left(\Gamma_{T}\right)$, so it is just a copy of $M_{T}$, and $F^{(j)}$ is isometric to $\mathcal{H} / \Lambda$. Now with $\Delta_{0}$ as in Proposition 1.1 , for $n \geq 1$ let

$$
\Delta_{n}=\left\langle\Delta_{0}, \Gamma_{T}^{(1)}, \ldots, \Gamma_{T}^{(n)}\right\rangle
$$

(In [3], $\Delta_{0}$ is denoted as $\Gamma_{S}$ and $\Delta_{n}$ as $\Gamma_{-}^{(n)}$.) The consequence of Propositions 3.10 and 3.12 of [3] below shows that $C\left(\Delta_{n}\right)$ is a geometric model for $B^{3}-T_{n}$.

Proposition 1.2. For each $n \in \mathbb{N}$ there is a homeomorphism $f_{n}: B^{3}-T_{n} \rightarrow$ $C\left(\Delta_{n}\right)$. Moreover, the natural map $C\left(\Delta_{n-1}\right) \rightarrow C\left(\Delta_{n}\right)$ is an isometric embedding, and there is another, $\iota_{n}: C\left(\Gamma_{T}\right) \rightarrow C\left(\Delta_{n}\right)$ factoring through an isometry $C\left(\Gamma_{T}\right) \rightarrow$ $C\left(\Gamma_{T}^{(i)}\right)$, such that for $n>1$ the following diagrams commute.
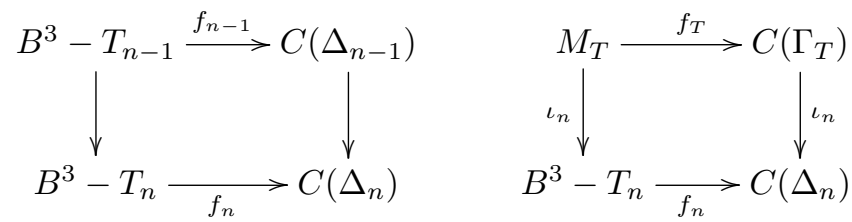

This also holds for $n=1$, taking $f_{0} \doteq f_{S}: M_{S} \rightarrow C\left(\Delta_{0}\right)$ at the top left.

The natural map $F^{(j)} \rightarrow f_{n}\left(S^{(j)}-\partial T_{j}\right)$ is an isometry onto a totally geodesic surface in $C\left(\Delta_{n}\right)$ when $0 \leq j \leq n$; in particular, $F^{(n)}$ is isometric to $\partial C\left(\Delta_{n}\right)$.

Our final task in comprehensively describing the $T_{n}$ is to translate the tangle endpoint labeling to the geometric setting, yielding a labeling of cusps of $F^{(n)}$, or equivalently, of parabolic conjugacy classes in $\Lambda^{(n)}$. We begin below by listing representatives for the parabolic conjugacy classes in $\Lambda$ as words in $\Gamma_{S}$ and $\Gamma_{T}$.

$$
\begin{aligned}
& \mathrm{p}_{1}=\mathrm{s}^{-1}=\mathrm{f}^{-1} \\
& \mathrm{p}_{2}=\mathrm{stst}^{-2}=\mathrm{fg}^{-1} \mathrm{f}^{-1} \mathrm{~h}^{-1} \mathrm{~g} \\
& \mathrm{p}_{3}=(\mathrm{tst}) \mathrm{s}^{-1}(\mathrm{tst})^{-1}=\left(\mathrm{h}^{-1} \mathrm{fg}\right)^{-1} \mathrm{~g}^{-1}\left(\mathrm{~h}^{-1} \mathrm{fg}\right), \\
& \mathrm{p}_{4}=\mathrm{p}_{1} \mathrm{p}_{2} \mathrm{p}_{3}^{-1}
\end{aligned}
$$

A calculation shows that

$$
\mathrm{p}_{1}=\left(\begin{array}{cc}
1 & 0 \\
1 & 1
\end{array}\right) \quad \mathrm{p}_{2}=\left(\begin{array}{cc}
-1 & 5 \\
0 & -1
\end{array}\right) \quad \mathrm{p}_{3}=\left(\begin{array}{cc}
-14 & 25 \\
-9 & 16
\end{array}\right) \quad \mathrm{p}_{4}=\left(\begin{array}{ll}
29 & -45 \\
20 & -31
\end{array}\right)
$$

From Lemma 2.4 of [3] we obtain the next proposition.

Proposition 1.3. For any $n \in \mathbb{N}, j \leq n$, and $k \in\{1,2,3,4\}$, the parabolic conjugacy class in $\Lambda^{(j)}$ which corresponds to the point labeled $k$ in $S^{(j)}$ is represented by $\mathrm{p}_{k}^{(j)}=\mathrm{c}^{-2 j} \mathrm{p}_{k} \mathrm{c}^{2 j}$. Also $\Lambda^{(j)}$ is generated by any three of the $\mathrm{p}_{k}^{(j)}$ 's.

We finish by giving a geometric model for the mutation with a hidden extension. The result below follows from Proposition 1.3 above and Lemma 5.5 of [3]. 
Lemma 1.4. Let $\mathrm{m}_{1}=\left(\begin{array}{cc}-3 & 5 \\ -2 & 3\end{array}\right)$. For each $n \geq 0, \mathrm{~m}_{1}^{(n)} \doteq \mathrm{c}^{-2 n} \mathrm{~m}_{1} \mathrm{c}^{2 n}$ normalizes $\Lambda^{(n)}$ and induces a cycle representation $(13)(24)$ on the the four cusps of $F^{(n)}$, where each cusp is numbered according to its corresponding parabolic isometry $\mathrm{p}_{j}^{(n)}$.

1.2. The proof of existence. The key new tool we need to prove Theorem 1.8 is a discrete group containing both $\Delta_{n}$, with finite index, and also the isometry $\mathrm{m}_{1}$ that induces the mutation (13)(24). (The group $G_{m+n}$ of [3, Lemma 6.2] plays this role for the group $\Gamma_{m+n}$ uniformizing $S^{3}-L_{m+n}$, by [3, Prop. 6.3].) We will use this with a standard argument to show there is a hidden extension.

As in Definitions 6.1 of [3], let $\mathcal{B}_{0}$ be the open half-ball in the upper half-space model of $\mathbb{H}^{3}$ bounded by the Euclidean hemisphere of unit radius centered at $0 \in \mathbb{C}$ and, for $k \in \mathbb{N}$, let $\mathcal{B}_{k}$ be the Euclidean translate of $\mathcal{B}_{0}$ centered at $k(-i \sqrt{2})$, where $i$ is the imaginary unit. For complex numbers $z$ and $w$, refer by $z \mathcal{H}+w$ to the geodesic plane $(z \mathbb{R}+w) \times(0, \infty)$.

Definition 1.5. For an integer $n \geq 0$, define $\mathcal{Q}_{n}$ to be the polyhedron of $\mathbb{H}^{3}$ bounded by $\mathcal{H}+i / 2, i \mathcal{H}, i \mathcal{H}+1 / 2$, and $\partial \mathcal{B}_{k}$ for $k \in\{0,1, \ldots, n\}$. Further define:

(1) $\mathrm{f}_{0}$ by first reflecting in $i \mathcal{H}$ and then in $i \mathcal{H}+1 / 2$;

(2) $b_{0}$ by first reflecting in $\mathcal{H}+i / 2$ and then in $\partial \mathcal{B}_{0}$; and

(3) for $k \geq 0, \mathrm{a}_{k}$ by reflecting in $i \mathcal{H}+1 / 2$ and then in $\partial \mathcal{B}_{k}$.

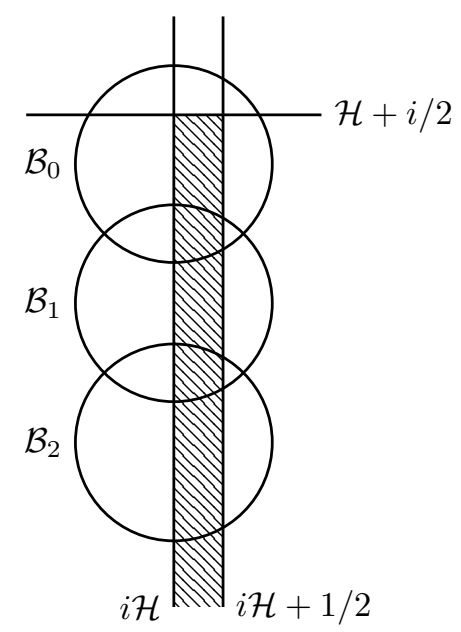

FiguRE 3. Bounding hyperplanes for $\mathcal{Q}_{2}$ viewed from above.

As defined, we have

$$
\mathrm{f}_{0}=\left(\begin{array}{ll}
1 & 1 \\
0 & 1
\end{array}\right) \quad \mathrm{b}_{0}=\left(\begin{array}{ll}
0 & i \\
i & 1
\end{array}\right) \quad \mathrm{a}_{0}=\left(\begin{array}{ll}
0 & -1 \\
1 & -1
\end{array}\right) \quad \mathrm{a}_{1}=\left(\begin{array}{cc}
-i \sqrt{2} & 1+i \sqrt{2} \\
1 & -1+i \sqrt{2}
\end{array}\right) .
$$

In particular, we see that $\left\langle\mathrm{f}_{0}, \mathrm{a}_{0}\right\rangle=\mathrm{PSL}_{2}(\mathbb{Z})$ contains $\mathrm{m}_{1}$.

Lemma 1.6. For each integer $n \geq 0$, the orientation-preserving subgroup $H_{n}$ of the group generated by reflections in the faces of $\mathcal{Q}_{n}$ satisfies:

(1) $H_{n}$ is a Kleinian group generated by $\left\{\mathrm{f}_{0}, \mathrm{~b}_{0}, \mathrm{a}_{0}, \ldots, \mathrm{a}_{n}\right\}$.

(2) $\Delta_{n}<H_{2 n}$ with finite index. 
(3) The projection $\mathrm{c}^{-n}(\mathcal{H}) \rightarrow \mathbb{H}^{3} / H_{n}$ factors through an isometric embedding of $\mathcal{H} / \mathrm{PSL}_{2}(\mathbb{Z})$ onto $\partial C\left(H_{n}\right)$.

Proof. Clearly $\mathrm{f}_{0} \in H_{n}, \mathrm{~b}_{0} \in H_{n}$, and $\mathrm{a}_{i} \in H_{n}$ for $0 \leq i \leq n$. Let $\mathrm{r}^{\prime}$ denote the reflection across $i \mathcal{H}+1 / 2$. It is not hard to see that $\mathcal{Q}_{n} \cup \mathrm{r}^{\prime}\left(\mathcal{Q}_{n}\right)$ is a convex polyhedron in $\mathbb{H}^{3}$ with one face in each of $\mathcal{H}+i \sqrt{2}, i \mathcal{H}, i \mathcal{H}+1, \partial \mathcal{B}_{k}$, and $\partial \mathrm{r}^{\prime}\left(\mathcal{B}_{k}\right)$ for $k \in\{0,1, \ldots, n\}$. The following facts can be explicitly verified:

- The face in $\mathcal{H}+i \sqrt{2}$ meets those in $i \mathcal{H}$ and $i \mathcal{H}+1$ at right angles, those in $\partial \mathcal{B}_{0}$ and $\mathbf{r}^{\prime}\left(\partial \mathcal{B}_{0}\right)$ at an angle of $\pi / 3$, and no others. The product $\mathrm{a}_{0} \mathrm{~b}_{0}$ rotates by $\pi$ about an axis that bisects this face, preserving it.

- The face in $i \mathcal{H}$ meets each of those in $\partial \mathcal{B}_{k}$ at an angle of $\pi / 2$, and none of those in $r^{\prime}\left(\partial \mathcal{B}_{j}\right)$. The element $\mathrm{f}_{0}$ takes this face to the one in $i \mathcal{H}+1$.

- The face in $\partial \mathcal{B}_{k}$ shares an edge with the face in $r^{\prime}\left(\partial \mathcal{B}_{k^{\prime}}\right)$ if and only if $k=k^{\prime}$; in this case at an angle of $2 \pi / 3$. The element $\mathrm{a}_{k}$ takes the latter to the former. The faces in $\partial \mathcal{B}_{k}$ and $\partial \mathcal{B}_{k-1}$ meet at an angle of $\pi / 2$ for $k>0$; likewise those in $\partial \mathcal{B}_{k}$ and $\partial \mathcal{B}_{k+1}$ for $k<n$; and $\partial \mathcal{B}_{k} \cap \partial \mathcal{B}_{k^{\prime}}=\emptyset$ for $k^{\prime} \notin\{k-1, k, k+1\}$.

Hence, $\left\{\mathrm{f}_{0}, \mathrm{a}_{0} \mathrm{~b}_{0}, \mathrm{a}_{0}, \ldots, \mathrm{a}_{n}\right\}$ is a face-pairing for $\mathcal{Q}_{n} \cup \mathrm{r}^{\prime}\left(\mathcal{Q}_{n}\right)$. Poincare's polyhedron theorem implies that this set of isometries generates a discrete group whose fundamental domain is $\mathcal{Q}_{n} \cup \mathrm{r}^{\prime}\left(\mathcal{Q}_{n}\right)$. By construction, this group is contained in $H_{n}$. It is equal to $H_{n}$ because their fundamental domains have the same volume.

The numbered formulas (8) and (9) above Proposition 6.3 of [3] express the generators of $\Delta_{0}$ in terms of $a_{0}, b_{0}$, and $f_{0}$ and they express $\Gamma_{T_{0}}$ in terms of $a_{0}, a_{1}$, and $\mathrm{f}_{0}$. Therefore, $\Delta_{0}<H_{n}$ and $\Gamma_{T_{0}}<H_{n}$. It can be verified directly that, for every $k, \mathrm{c}^{-1} \mathrm{a}_{k} \mathrm{c}=\mathrm{a}_{k+1}$ and that $\mathrm{c}$ commutes with $\mathrm{f}_{0}$. It follows that $\mathrm{c}^{-k} \Gamma_{T_{0}} \mathrm{c}^{k}<H_{n}$ for all $k \leq n-1$. Moreover, the second paragraph of the proof of [3, Proposition 6.3] expresses the generators of $\mathrm{c}^{-2} \bar{\Gamma}_{T_{0}} \mathrm{c}^{2}$ in terms of $\mathrm{a}_{1}, \mathrm{a}_{2}$, and $\mathrm{f}_{0}$. So, if $n \geq 2$, this group is also in $H_{n}$. Now, by definition, we have $\Delta_{n}<H_{2 n}$.

The polyhedron $P_{n}$ of [3, Lemma 6.2] consists of points $(z, t) \in \mathcal{Q}_{n}$ such that the imaginary coordinate of $z$ is at least $-n \sqrt{2}$. Every face of $P_{n}$ is a face of $\mathcal{Q}_{n}$ except the unique face $\mathcal{F}$ of $P_{n}$ contained in $\mathcal{H}-n \cdot i \sqrt{2}$. The face $\mathcal{F}$ is orthogonal to $\partial \mathcal{B}_{n}, i \mathcal{H}$, and $i \mathcal{H}+1 / 2$ and does not meet any other bounding hyperplanes of $\mathcal{Q}_{n}$. This means that a single face of $P_{n} \cup \mathrm{r}^{\prime}\left(P_{n}\right)$ contains $\mathcal{F}$, meeting only the bounding hyperplanes $i \mathcal{H}, i \mathcal{H}+1, \partial \mathcal{B}_{n}$, and $\mathrm{r}^{\prime}\left(\partial \mathcal{B}_{n}\right)$. Moreover, these intersections are all orthogonal, so $\mathcal{F}$ projects to the sole totally geodesic boundary component of the orbifold $\left(P_{n} \cup \mathrm{r}^{\prime}\left(P_{n}\right)\right) / H_{n}$.

We claim that $\left(P_{n} \cup \mathrm{r}^{\prime}\left(P_{n}\right)\right) / H_{n}=C\left(H_{n}\right)$. We first show that $P_{n} \cup \mathrm{r}^{\prime}\left(P_{n}\right)$ is contained in the convex hull of the limit set of $H_{n}$, which implies that $\left(P_{n} \cup\right.$ $\left.\mathrm{r}^{\prime}\left(P_{n}\right)\right) / H_{n}$ is contained in the convex core. Inspecting Figures 3 and 4 in [3], one observes that $P_{n} \cup \mathrm{r}^{\prime}\left(P_{n}\right)$ is contained in the union $\mathcal{P}_{1} \cup \bigcup_{k=0}^{n-1} \mathrm{c}^{-k}\left(\mathcal{P}_{2}\right)$, where $\mathcal{P}_{1}$ and $\mathcal{P}_{2}$ are the regular ideal octahedron and right angled ideal cuboctahedron described in Corollaries 2.2 and 2.3 of [3]. Both $\mathcal{P}_{1}$ and $\mathcal{P}_{2}$ are the convex hulls of their ideal points, and each of these is a parabolic fixed point of $\Delta_{0}$ or $\Gamma_{T_{0}}$, respectively. (One can show this directly, or appeal to the third-from-last paragraph of the proof of [3, Lemma 2.1].) Since each parabolic fixed point of a Kleinian group lies in its limit set, it follows that $\mathcal{P}_{1} \cup \bigcup_{k=0}^{n-1} \mathrm{c}^{-k}\left(\mathcal{P}_{2}\right)$ is in the convex hull of the limit set of $H_{n}$. As a subset, $P_{n} \cup \mathrm{r}^{\prime}\left(P_{n}\right)$ shares this property. On the other hand, the penultimate 
paragraph of [3, proof of Lemma 2.1] shows that $\left(P_{n} \cup \mathrm{r}^{\prime}\left(P_{n}\right)\right) / H_{n}$ contains $C\left(H_{n}\right)$ and this proves our claim.

By the above, $\mathrm{c}^{-2 n}(\mathcal{H})$ projects to $\partial C\left(H_{2 n}\right)$ under the quotient map $\mathbb{H}^{3} \rightarrow$ $\mathbb{H}^{3} / H_{2 n}$. By Proposition 1.2, the same plane projects to $\partial C\left(\Delta_{n}\right)$ under $\mathbb{H}^{3} \rightarrow$ $\mathbb{H}^{3} / \Delta_{n}$. It follows that the orbifold covering map $\mathbb{H}^{3} / \Delta_{n} \rightarrow \mathbb{H}^{3} / H_{2 n}$ restricts to one $C\left(\Delta_{n}\right) \rightarrow C\left(H_{2 n}\right)$. Since these both have finite volume, the map is finite-to-one, and hence $\Delta_{n}$ has finite index in $H_{2 n}$.

Among all bounding hyperplanes of $\mathcal{Q}_{n} \cup \mathrm{r}^{\prime}\left(\mathcal{Q}_{n}\right)$, only $i \mathcal{H}, i \mathcal{H}+1, \partial \mathcal{B}_{n}$, and $\mathrm{r}^{\prime}\left(\partial \mathcal{B}_{n}\right)$ meet the hyperplane $\mathcal{H}-n \cdot i \sqrt{2}$. Each of these intersections is a right angle. Thus, $\mathcal{F}$ is a quadrilateral and $\left\{a_{n}, f_{0}\right\}$ is an edge pairing for $\mathcal{F}$. This implies that $\mathcal{F} /\left\langle\mathrm{a}_{n}, \mathrm{f}_{0}\right\rangle$ is the boundary of $\left(P_{n} \cup \mathrm{r}^{\prime}\left(P_{n}\right)\right) / H_{n}$.

We mentioned above that $\mathrm{c}^{-n} \mathrm{a}_{0} \mathrm{c}^{n}=\mathrm{a}_{n}$ and $\mathrm{f}_{0} \mathrm{c}=\mathrm{cf}_{0}$, so $\left\langle\mathrm{a}_{n}, \mathrm{f}_{0}\right\rangle=\mathrm{c}^{-n}\left\langle\mathrm{a}_{0}, \mathrm{f}_{0}\right\rangle \mathrm{c}^{n}$. Therefore, the projection $\mathcal{H}-n \cdot i \sqrt{2}=\mathrm{c}^{-n}(\mathcal{H}) \rightarrow \mathbb{H}^{3} / H_{n}$ factors through an isometric embedding of $\mathcal{H} / \mathrm{PSL}_{2}(\mathbb{Z})$.

We will use the following simple fact below, and several more times.

Fact 1.7. If $H$ has finite index in a non-elementary Kleinian group $G$ then the limit sets of $G$ and $H$ are equal, so the natural map $C(H) \rightarrow C(G)$ is an orbifold cover.

Theorem 1.8. For $n \in \mathbb{N}$, the mutation of $\partial\left(B^{3}-T_{n}\right)$ determined by $(13)(24)$ has a hidden extension over a cover of $B^{3}-T_{n}$ and for any $m \in \mathbb{N}$, taking $T_{n} \subset L_{m+n}$, a hidden extension over a cover of $S^{3}-L_{m+n}$.

Proof. As we mentioned in the introduction to this paper, we may view $\left(B^{3}, T_{n}\right)$ as a subset of $\left(S^{3}, L_{n+m}\right)$. (For more rigor, compare the definitions at the beginning of this section with [3, Definitions 3.8].) Here it is bounded by the sphere $S^{(n)}$, with the mirror image $\left(B^{3}, \bar{T}_{m}\right)$ of $T_{m}$ on the other side. If the mutation (13)(24) extended over $B^{3}-T_{n}$, then $S^{3}-L_{n+m}$ would be homeomorphic to its mutant by $(13)(24)$ along $S^{(n)}$. By Mostow-Prasad rigidity, these two links would be isometric, but by Theorem 2 of [3] they are not. (In the notation of that result, $L_{m+n}=L_{(0, \ldots, 0)}$ and its mutant is $L_{(0, \ldots, 1, \ldots, 0)}$ with the sole "1" the $(m+1)$ th entry.) This also implies it does not extend over $S^{3}-L_{m+n}$.

However, because $\mathrm{m}_{1}^{(n)}$ lies in the finite extension $H_{2 n}$ of $\Delta_{n}$ it normalizes the normal core $\Omega_{n}$ of $\Delta_{n}$ in $H_{2 n}$ and determines a self-isometry $\tilde{\Psi}$ of $\mathbb{H}^{3} / \Omega_{n}$. This is a finite cover of $\mathbb{H}^{3} / \Delta_{n}$ which by Fact 1.7 above restricts to a cover $C\left(\Omega_{n}\right) \rightarrow C\left(\Delta_{n}\right)$.

In particular, the boundary of $C\left(\Omega_{n}\right)$ is totally geodesic. One component of $\partial C\left(\Omega_{n}\right)$ is the quotient of $\mathrm{c}^{-2 n}(\mathcal{H})$ by its stabilizer $\tilde{\Lambda}^{(n)}=\Omega_{n} \cap \Lambda^{(n)}$ in $\Omega_{n}$. Since $\mathrm{m}_{1}^{(n)}$ normalizes both $\Lambda^{(n)}$ and $\Omega_{n}$, it normalizes $\tilde{\Lambda}^{(n)}$ and determines an isometry of $\mathrm{c}^{-2 n}(\mathcal{H}) / \tilde{\Lambda}^{(n)}$ lifting the one determined by $\mathrm{m}_{1}^{(n)}$ on $\partial C\left(\Delta_{n}\right)$.

A completely analogous argument applies to $S^{3}-L_{m+n}$, replacing $\Delta_{n}$ by $\Gamma_{n}$ from Prop. 3.12 of [3] and $H_{2 n}$ by $G_{n}$ from Prop. 6.3 there.

\section{MAtChing COVERS}

In this section, we build an explicit hidden extension of the mutation (13)(24) of $\partial\left(B^{3}-T_{n}\right)$. To find an appropriate cover, we use the decomposition of $B^{3}-T_{n}$ along the spheres $S^{(j)}$ into one copy of $M_{S}$ and $n$ copies of $M_{T}$ and find appropriate covers of these pieces which glue together to give a cover of $B^{3}-T_{n}$ with the necessary properties. Figure 4 is a schematic depiction of how this will be done. 


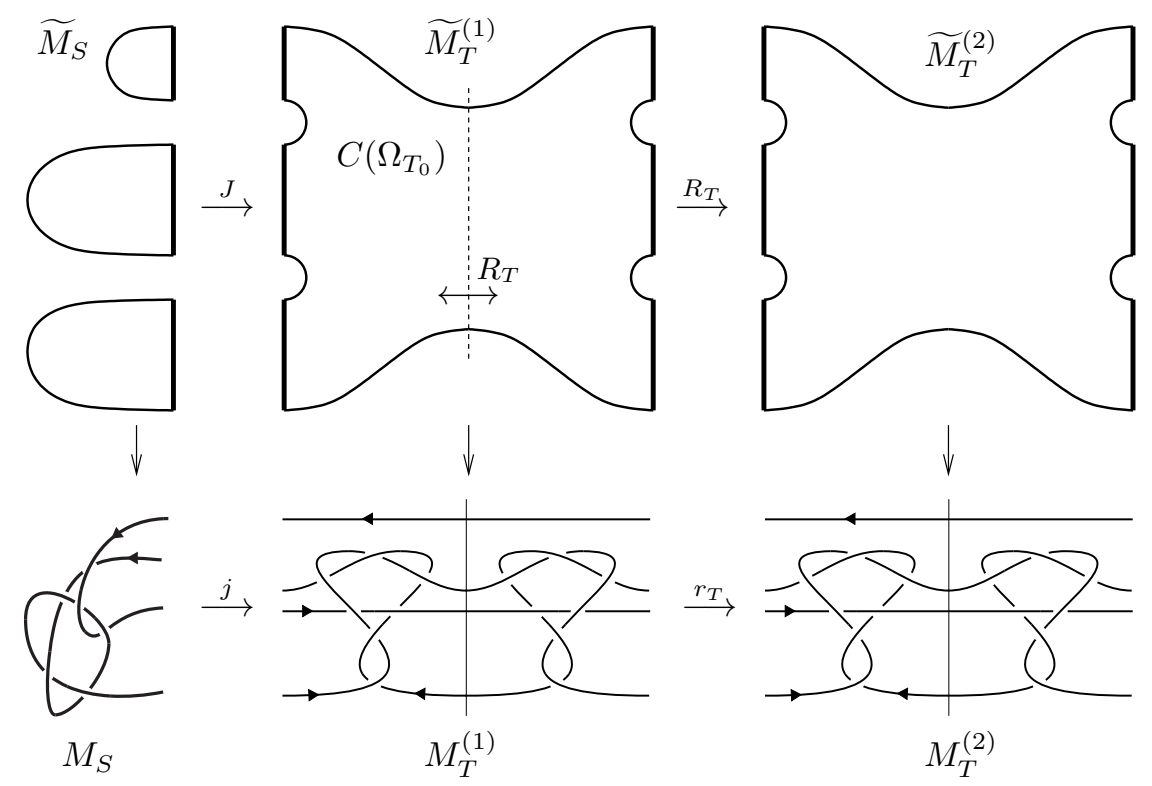

Figure 4. Assembling a cover of $\left(S^{2} \times I\right)-T_{2}$ that has a hidden extension of the mutation (13)(24).

For convenience, in this section we will supress the homeomorphisms $f_{S}, f_{T_{0}}$, and $f_{T}$ of Proposition 1.1 and simply make the identifications:

$$
M_{S}=C\left(\Delta_{0}\right) \quad M_{T_{0}}=C\left(\Gamma_{T_{0}}\right) \quad M_{T}=C\left(\Gamma_{T}\right) .
$$

Further, for $n \in \mathbb{N}$ and $1 \leq i \leq n$ we will identify the $i$ th copy $M_{T}^{(i)}$ of $\left(S^{2} \times I, T\right)$ in $\left(B^{3}, T_{n}\right)$ with $C\left(\Gamma_{T}^{(i)}\right)$ (compare Proposition 1.2).

To produce the covers $\widetilde{M}_{S}$ and the $\widetilde{M}_{T}^{(i)}$ of Figure 4 we will divide the orbifold $O_{n}=\mathbb{H}^{3} / H_{n}$ branched-covered by $B^{3}-T_{n}$ into pieces covered by $M_{S}$ and the $M_{T}^{(i)}$, then analyze the corresponding subgroups of $H_{n}$. For $M_{S}$ we use $\mathcal{Q}_{0}$ from

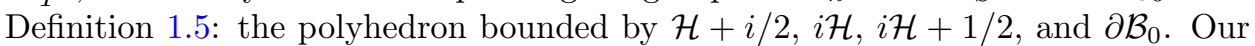
first lemma shows that the orientation-preserving subgroup $H_{0}$ of the reflection group generated by $\mathcal{Q}_{0}$ contains the group $\Delta_{0}=\Gamma_{S}$ uniformizing $M_{S}$. Our second uses $H_{0}$ and some elementary number theory to find a cover of $M_{S}$ with abundant symmetry.

We follow a similar strategy for $M_{T}$, producing a polyhedron $\mathcal{P}_{T_{0}}$ and a group $H_{T_{0}}<H_{n}$, which Lemma 2.3 shows contains the group $\Gamma_{T_{0}}$ uniformizing $M_{T_{0}}$. We will use the permutation representation of $H_{T_{0}}$ given by acting on left cosets of $\Gamma_{T_{0}}$ to find a cover of $M_{T_{0}}$ with a hidden extension of (13)(24). Doubling this cover across a boundary component yields the model $\widetilde{M}_{T}$ for the $\widetilde{M}_{T}^{(i)}$.

Lemma 2.1. The reflection group $H_{0}$ (recall Lemma 1.6) has the following additional properties:

(1) $H_{0}$ is a Kleinian group which contains $\Delta_{0}$ as a subgroup of index 12.

(2) $H_{0}=\left\langle a_{0}, b_{0}, f_{0} \mid a_{0}^{3}=b_{0}^{3}=\left(b_{0}^{-1} a_{0}\right)^{2}=\left(a_{0} f_{0}\right)^{2}=1\right\rangle$.

(3) $\operatorname{PSL}_{2}(\mathbb{Z})=\operatorname{Stab}_{H_{0}}(\mathcal{H})$. 
(4) The projection $\mathcal{H} \rightarrow \mathbb{H}^{3} / H_{0}$ factors through an isometric embedding of $\mathcal{H} / \mathrm{PSL}_{2}(\mathbb{Z})$ onto $\partial C\left(H_{0}\right)$.

Proof. As in Lemma 1.6, $r^{\prime}$ is the reflection through $i \mathcal{H}+1 / 2$. From the proof of Lemma 1.6, we know that the collection $\left\{\mathrm{a}_{0}, \mathrm{~b}_{0} \mathrm{a}_{0}, \mathrm{f}_{0}\right\}$ is a face-pairing for $\mathcal{Q}_{0} \cup$ $r^{\prime}\left(\mathcal{Q}_{0}\right)$. So the Poincaré polyhedron theorem gives the presentation above. Aside from the fact that $\left[H_{0}, \Delta_{0}\right]=12$, the rest of the lemma follows from the special case $n=0$ in Lemma 1.6. But, if we compare the volume of a regular ideal octahedron in $\mathbb{H}^{3}$ with the volume of $P_{0}$, we see that $\left[H_{0}, \Delta_{0}\right]=12$.

Lemma 2.2. There is an index five subgroup $\Omega_{0}<\Delta_{0}$ which is normal in $H_{0}$. Define $\Lambda_{0}=\Omega_{0} \cap \Lambda$.

(1) $\left[\Lambda: \Lambda_{0}\right]=5$,

(2) $\mathrm{p}_{2}, \mathrm{p}_{4} \in \Lambda_{0}$, and

(3) $\mathrm{p}_{1}$ and $\mathrm{p}_{3}$ project to generators of $\Lambda / \Lambda_{0}$.

Proof. In the ring of Gaussian integers $5=(1+2 i)(1-2 i)$. So, restricting the map $\mathbb{Z}[i] \rightarrow \mathbb{Z}[i] /(1+2 i)$ to $\mathbb{Z}$ gives a ring epimorphism $\mathbb{Z} \rightarrow \mathbb{Z}[i] /(1+2 i)$. The quotient ring $\mathbb{Z}[i] /(1+2 i)$ is isomorphic to $\mathbb{Z} / 5 \mathbb{Z}$ and we obtain a group epimorphism $\mathrm{PSL}_{2}(\mathbb{Z}[i]) \rightarrow \mathrm{PSL}_{2}(\mathbb{Z} / 5 \mathbb{Z})$ which restricts to an epimorphism $\mathrm{PSL}_{2}(\mathbb{Z}) \rightarrow$ $\mathrm{PSL}_{2}(\mathbb{Z} / 5 \mathbb{Z})$. Since $\mathrm{PSL}_{2}(\mathbb{Z})<H_{0}<\mathrm{PSL}_{2}(\mathbb{Z}[i])$, the restriction to $H_{0}$ is also onto and the kernel $\Omega_{0}$ of this map has index $\left|\mathrm{PSL}_{2}(\mathbb{Z} / 5 \mathbb{Z})\right|=60$ in $H_{0}$.

Using the explicit descriptions of $\mathrm{s}$ and $\mathrm{t}$ from Section 1 , we see that $\Delta_{0}$ maps onto the parabolic subgroup $\left\{\left(\begin{array}{ll}1 & 0 \\ * & 1\end{array}\right)\right\}$ of $\mathrm{PSL}_{2}(\mathbb{Z}[i] /(1+2 i))$ which has order 5 . Hence, $\Delta_{0} \cap \Omega_{0}$ has index five in $\Delta_{0}$. Since $\left[H_{0}: \Delta_{0}\right]=12$, it follows that $\left[H_{0}: \Delta_{0} \cap \Omega_{0}\right]=$ 60. Therefore, $\Delta_{0}$ contains $\Omega_{0}$.

Similarly, the explicit descriptions of the $\mathrm{p}_{j}$ 's from Section 1 show that $\Lambda$ maps onto this same parabolic subgroup and $\left[\Lambda: \Lambda_{0}\right]=5$. The final assertion is also immediate from these descriptions.

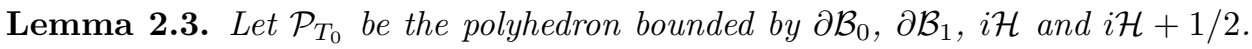
The orientation-preserving subgroup $H_{T_{0}}$ of the group generated by reflections in the sides of $\mathcal{P}_{T_{0}}$ is a Kleinian group such that

(1) $H_{T_{0}}=\left\langle a_{0}, a_{1}, f_{0} \mid a_{0}^{3}=a_{1}^{3}=1,\left(a_{0} a_{1}^{-1}\right)^{2}=\left(a_{0} f_{0}\right)^{2}=\left(a_{1} f_{0}\right)^{2}=1\right\rangle$,

(2) $\partial C\left(H_{T_{0}}\right)$ consists of a pair of totally geodesic surfaces,

(3) $\mathrm{PSL}_{2}(\mathbb{Z})$ and $\Gamma_{T_{0}}$ are subgroups of $H_{T_{0}}$, and

(4) $\left[H_{T_{0}}: \Gamma_{T_{0}}\right]=\left[\operatorname{Stab}_{H_{T_{0}}}(\mathcal{H}): \Lambda\right]=12$.

Proof. First, recall from just before Lemma 1.6 and in the proof of Lemma 1.6 that Claim (3) has already been established.

Now, for visual intuition, compare $\mathcal{P}_{T_{0}}$ with the right-angled ideal cuboctahedron $\mathcal{P}_{2}$. The intersection $\mathcal{P}_{T_{0}} \cap \mathcal{P}_{2}$ is the portion of $\mathcal{P}_{T_{0}}$ which lies between $\mathcal{H}$ and $\mathcal{H}-$ $i \sqrt{2}$. The intersection $\mathcal{P}_{T_{0}} \cap \mathcal{P}_{2}$ has two additional faces contained in $\mathcal{H}$ and $\mathcal{H}-i \sqrt{2}$ and a single ideal vertex at $\infty$. These additional faces are either perpendicular to or disjoint from those of $\mathcal{P}_{T_{0}}$.

Let $\mathcal{F}$ be the face of $\mathcal{P}_{T_{0}} \cap \mathcal{P}_{2}$ contained in $i \mathcal{H}, \mathcal{A}_{0}$ its face in $\partial \mathcal{B}_{0}$, and $\mathcal{A}_{1}$ the face in $\partial \mathcal{B}_{1}$. Let $r^{\prime}$ denote reflection across $i \mathcal{H}+1 / 2$. A fundamental domain for $H_{T_{0}}$ is the union $\mathcal{P}_{T_{0}} \cup \mathrm{r}^{\prime}\left(\mathcal{P}_{T_{0}}\right)$. By construction, $\mathrm{a}_{0}, \mathrm{a}_{1}, \mathrm{f}_{0} \in H_{T_{0}}$ and these isometries determine a face-pairing for this fundamental domain. In particular, $\mathrm{a}_{0}\left(\mathrm{r}^{\prime}\left(\mathcal{A}_{0}\right)\right)=\mathcal{A}_{0}, \mathrm{a}_{1}\left(\mathrm{r}^{\prime}\left(\mathcal{A}_{1}\right)\right)=\mathcal{A}_{1}$, and $\mathrm{f}_{0}(\mathcal{F})=\mathrm{r}^{\prime}(\mathcal{F})$. Thus, $H_{T_{0}}$ is generated by $\mathrm{a}_{0}, \mathrm{a}_{1}$, and $\mathrm{f}_{0}$. Upon noting that, for each $i \in\{0,1\}, \mathcal{A}_{i}$ intersects $\mathcal{F}$ and $\mathcal{A}_{1-i}$ 
perpendicularly and $\mathbf{r}^{\prime}\left(\mathcal{A}_{i}\right)$ at an angle of $2 \pi / 3$, the above presentation comes from the usual edge-cycle relations.

We claim that $C\left(H_{T_{0}}\right)$ is the quotient of $\mathcal{Q}_{T_{0}} \doteq \mathcal{P}_{2} \cap\left(\mathcal{P}_{T_{0}} \cup \mathrm{r}\left(\mathcal{P}_{T_{0}}\right)\right)$ by the facepairing isometries, with totally geodesic boundary. As remarked above, the faces $\mathcal{Q}_{T_{0}} \cap \mathcal{H}$ and $\mathcal{Q}_{T_{0}} \cap(\mathcal{H}-i \sqrt{2})$ of $\mathcal{Q}_{T_{0}}$ intersect the others perpendicularly, so $\mathcal{Q}_{T_{0}}$ projects under the quotient map $\mathbb{H}^{3} / H_{T_{0}}$ to a suborbifold with totally geodesic boundary. This is contained in $C\left(H_{T_{0}}\right)$, as $\mathcal{Q}_{T_{0}} \subset \mathcal{P}_{2}$, which by Corollary 2.3 of [3] is contained in the convex hull of the limit set of $\Gamma_{T_{0}}$ which is contained in the convex hull of the limit set of $H_{T_{0}}$. Arguing as in the proof of Lemma 2.1 of [3] gives the reverse inclusion and hence the claim.

That $\left[H_{T_{0}}: \Gamma_{T_{0}}\right]=12$ follows from volume considerations. Note that $\mathrm{a}_{0}$ and $\mathrm{a}_{1}$ each preserve $\mathcal{P}_{2}$ and fix the point $\left(A_{0} \cap A_{1}\right) \cap\left(\mathrm{r}\left(A_{0}\right) \cap \mathrm{r}\left(A_{1}\right)\right)$; thus the group they generate has these properties as well. Since $\mathcal{Q}_{T_{0}}$ contains a neighborhood in $\mathcal{P}_{2}$ of its ideal vertex $\infty,\left\langle\mathrm{a}_{0}, \mathrm{a}_{1}\right\rangle$ acts freely on the set ideal vertices of $\mathcal{P}_{2}$. It is not hard to show directly that this action is transitive. Since $\mathcal{P}_{2}$ has twelve ideal vertices, its volume is twelve times that of $\mathcal{Q}_{T_{0}}$. Since these are fundamental domains for $M_{T_{0}}$ and $C\left(H_{T_{0}}\right)$ the associated cover has degree twelve.

Recall from above that the faces $\mathcal{Q}_{T_{0}} \cap \mathcal{H}$ and $\mathcal{Q}_{T_{0}} \cap(\mathcal{H}-i \sqrt{2})$ of $\mathcal{Q}_{T_{0}}$ project to $\partial C\left(H_{T_{0}}\right)$. The face-pairings of $\mathcal{Q}_{T_{0}}$ induce edge-pairings on these faces, and one checks directly that each face has its edges identified to each other. It follows that $\partial C\left(H_{T_{0}}\right)$ has two components. Since $\partial M_{T_{0}}$ also has two components, each component of $\partial M_{T_{0}}$ covers twelve-to-one. Since $\partial M_{T_{0}}$ has a component isometric to $F^{(0)},\left[\operatorname{Stab}_{H_{T_{0}}}(\mathcal{H}): \Lambda\right]=12$.

Lemma 2.4. There is a homomorphism $\phi: H_{T_{0}} \rightarrow S_{12}$ determined by

$$
\begin{aligned}
& \phi\left(\mathrm{a}_{0}\right)=\left(\begin{array}{lll}
1 & 5 & 9
\end{array}\right)\left(\begin{array}{lll}
2 & 6 & 10
\end{array}\right)\left(\begin{array}{lll}
3 & 7 & 11
\end{array}\right)\left(\begin{array}{lll}
4 & 8 & 12
\end{array}\right) \\
& \phi\left(\mathrm{a}_{1}\right)=\left(\begin{array}{lll}
1 & 8 & 10
\end{array}\right)\left(\begin{array}{llll}
2 & 7 & 9
\end{array}\right)\left(\begin{array}{lll}
3 & 6 & 12
\end{array}\right)\left(\begin{array}{lll}
4 & 5 & 11
\end{array}\right) \\
& \phi\left(f_{0}\right)=\left(\begin{array}{lllll}
1 & 5 & 11 & 10 & 3
\end{array}\right)\left(\begin{array}{lllll}
2 & 7 & 6 & 8 & 12
\end{array}\right)
\end{aligned}
$$

It has the following properties.

(1) $\left|\phi\left(H_{T_{0}}\right)\right|=660$,

(2) $\phi\left(\Gamma_{T_{0}}\right)=\langle\phi(\mathrm{h}), \phi(\mathrm{f})\rangle \cong \mathbb{Z}_{11} \rtimes \mathbb{Z}_{5}$, and

(3) $\phi(\Lambda)=\langle\phi(\mathrm{f})\rangle=\phi\left(\Gamma_{T_{0}}\right) \cap \phi\left(\mathrm{m}_{1} \Gamma_{T_{0}} \mathrm{~m}_{1}^{-1}\right)$ is the largest subgroup of $\phi\left(\Gamma_{T_{0}}\right)$ normalized by $\phi\left(\mathrm{m}_{1}\right)$.

Remark 2.5. The homomorphism $\phi$ above is the permutation representation of $H_{T_{0}}$ given by its action on the left cosets of $\Gamma_{T_{0}}$. This fact is not needed in the proof below or the rest of the paper.

Proof. That $\phi$ is a homomorphism follows from the presentation for $H_{T_{0}}$ given in Lemma 2.3. Our expressions for $\mathrm{a}_{0}, \mathrm{a}_{1}, \mathrm{f}_{0}, \mathrm{f}, \mathrm{g}, \mathrm{h}$, and $\mathrm{m}_{1}$ as matrices make it easy to verify the equalities

$$
\begin{array}{rlrl}
\mathrm{f} & =\mathrm{a}_{0} \mathrm{f}_{0} \mathrm{a}_{0}^{-1} & \mathrm{~g} & =\left(\mathrm{a}_{0}^{-1} \mathrm{a}_{1}\right) \mathrm{f}_{0}^{-1}\left(\mathrm{a}_{0}^{-1} \mathrm{a}_{1}\right)^{-1} \\
\mathrm{~h} & =\mathrm{a}_{1} \mathrm{a}_{0} \mathrm{f}_{0}^{-1} \mathrm{a}_{1} & \mathrm{~m}_{1} & =\left(\mathrm{f}_{0} \mathrm{a}_{0}^{-1}\right)^{2} \mathrm{f}_{0}^{-1} .
\end{array}
$$

This gives

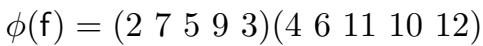

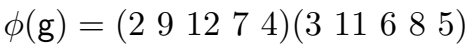

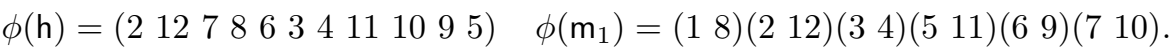


We see that $\phi\left(\Gamma_{T_{0}}\right)=\langle\phi(\mathrm{h}), \phi(\mathrm{f})\rangle \cong \mathbb{Z}_{11} \rtimes \mathbb{Z}_{5}$, because $\phi(\mathrm{g})=\phi\left(\mathrm{fh}^{-1}\right)$ and $\phi\left(\mathrm{fhf}^{-1}\right)=\phi\left(\mathrm{h}^{4}\right)$.

Under the action of $H_{T_{0}}$ on $\mathbb{Z}_{12}$ given by $\phi, \Gamma_{T_{0}}$ is a subgroup of $\operatorname{Stab}_{H_{T_{0}}}(1)$. We claim that, in fact, these groups are equal. Let

$$
\begin{array}{ll}
C=\{1,5,9\} & D=\{8,2,11\} \\
E=\{3,6,12\} & F=\{4,7,10\}
\end{array}
$$

and observe that $\phi\left\langle\mathrm{a}_{0}, \mathrm{a}_{1}\right\rangle$ preserves the triples $C, D, E$, and $F$. This gives a homomorphism $\psi:\left\langle\mathrm{a}_{0}, \mathrm{a}_{1}\right\rangle \rightarrow S_{4}$ with

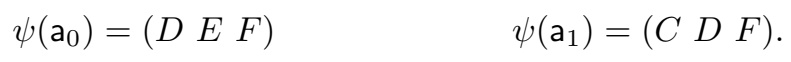

Since these two elements generate $A_{4}$ we have that the image of $\psi$ is the order 12 group $A_{4}$. The group $\left\langle\mathrm{a}_{0}, \mathrm{a}_{1}\right\rangle$ also acts by isometry on the polyhedron $\mathcal{P}_{2}$ and acts freely and transitively on its set of ideal vertices. Hence $\left|\left\langle\mathrm{a}_{0}, \mathrm{a}_{1}\right\rangle\right|=12$ and $\psi:\left\langle\mathrm{a}_{0}, \mathrm{a}_{1}\right\rangle \rightarrow A_{4}$ is an isomorphism.

Since $\left|\left\langle a_{0}, a_{1}\right\rangle\right|=12$ and $\Gamma_{T_{0}}$ is torsion-free, the elements of $\left\langle a_{0}, a_{1}\right\rangle$ make up a complete set of representatives for the left cosets of $\Gamma_{T_{0}}$. If $k \in \operatorname{Stab}_{H_{T_{0}}}$ (1) then $\mathrm{k}=\mathrm{an}$, where $\mathrm{a} \in\left\langle\mathrm{a}_{0}, \mathrm{a}_{1}\right\rangle$ and $\mathrm{n} \in \Gamma_{T_{0}}$. Then

$$
\phi(\mathrm{a}) \cdot 1=\phi(\mathrm{a}) \phi(\mathrm{n}) \cdot 1=\phi(\mathrm{k}) \cdot 1=1
$$

and so we must also have $\psi(\mathrm{a}) \cdot C=C$. If we list the elements of $A_{4}$, we see that the only possibilities for a are the identity or $\mathrm{a}_{0}^{ \pm 1}$. Since $\mathrm{a}_{0}^{ \pm 1}$ do not fix 1 , we must have $\mathrm{k} \in \Gamma_{T_{0}}$ as claimed.

We know now that $\operatorname{ker} \phi<\Gamma_{T_{0}}$, which implies that the images of distinct left cosets of $\Gamma_{T_{0}}$ in $H_{T_{0}}$ have empty intersection in $\phi\left(H_{T_{0}}\right)$. Therefore, $\left|\phi\left(H_{T_{0}}\right)\right|=$ $55 \cdot 12=660$.

Our formulas for the $\mathrm{p}_{j}$ 's in terms of $\mathrm{f}, \mathrm{g}$, and $\mathrm{h}$ give $\phi\left(\mathrm{p}_{1}\right)=\phi\left(\mathrm{p}_{3}\right)=\phi\left(\mathrm{f}^{-1}\right)$ and $\phi\left(\mathrm{p}_{2}\right)=\mathrm{id}$, so $\phi(\Lambda)=\left\langle\phi\left(\mathrm{f}^{-1}\right)\right\rangle$. Since $\mathrm{m}_{1}$ normalizes $\Lambda, \phi\left(\mathrm{m}_{1}\right)$ normalizes $\phi(\Lambda)$; and indeed we have that $\phi\left(\mathrm{m}_{1} \mathrm{fm}_{1}^{-1}\right)=\phi\left(\mathrm{f}^{-1}\right)$. On the other hand, $\phi\left(\mathrm{m}_{1} \mathrm{hm}_{1}^{-1}\right)$ does not stabilize 1 while $\phi\left(\Gamma_{T_{0}}\right)$ does, so $\phi\left(\Gamma_{T_{0}}\right) \cap \phi\left(\mathrm{m}_{1} \Gamma_{T_{0}} \mathrm{~m}_{1}^{-1}\right)$ is properly contained in $\phi\left(\Gamma_{T_{0}}\right)$. This intersection contains $\phi(\Lambda)$, so since $\left|\phi\left(\Gamma_{T_{0}}\right)\right|=55$ the intersection equals $\phi(\Lambda)$. It moreover follows that $\phi(\Lambda)$ is the largest subgroup of $\phi\left(\Gamma_{T_{0}}\right)$ normalized by $\phi\left(\mathrm{m}_{1}\right)$.

Define $\Omega_{T_{0}}=\phi^{-1}(\phi \Lambda)$, and let $p_{T_{0}}: \mathbb{H}^{3} / \Omega_{T_{0}} \rightarrow \mathbb{H}^{3} / \Gamma_{T_{0}}$ be the corresponding cover. If we let $\theta: H_{0} \rightarrow \mathrm{PSL}_{2}(\mathbb{Z}[i] /(1+2 i))$ be the map used in the proof of Lemma 2.2 and identify $\theta(\Lambda)$ and $\phi(\Lambda)$ with the isomorphism $\phi f \mapsto\left(\begin{array}{ll}1 & 0 \\ 4 & 1\end{array}\right)$, we see that $\theta=\phi$. In particular, $\Lambda_{0}=\Lambda \cap \operatorname{ker} \phi$.

Lemma 2.6. The preimage of $p_{T_{0}}^{-1}\left(F^{(0)}\right)$ has three components. One is the inclusioninduced image of $F^{(0)}$ in $\mathbb{H}^{3} / \Omega_{T_{0}}$, which projects isometrically to $\mathbb{H}^{3} / \Gamma_{T_{0}}$. The other two are respectively isometric to $\mathrm{g}(\mathcal{H}) /\left(\mathrm{g} \Lambda_{0} \mathrm{~g}^{-1}\right)$ and $\mathrm{g}^{-1}(\mathcal{H}) /\left(\mathrm{g}^{-1} \Lambda_{0} \mathrm{~g}\right)$, each of which projects five-to-one into $\mathbb{H}^{3} / \Gamma_{T_{0}}$.

Proof. Lemma 2.4 implies that $\left[\Gamma_{T_{0}}: \Omega_{T_{0}}\right]=11$. Fact 1.7 implies that $p_{T_{0}}$ restricts to a covering map $C\left(\Omega_{T_{0}}\right) \rightarrow M_{T_{0}}$. By definition of $\Omega_{T_{0}}$, $\Lambda$ is a subgroup of $\Omega_{T_{0}}$ and corresponds to a component of $\partial C\left(\Omega_{T_{0}}\right)$ that covers $\partial_{-} M_{T_{0}}$ one-to-one.

To understand the entire preimage $p_{T_{0}}^{-1}\left(\partial_{-} M_{T_{0}}\right)$ we pass to the cover $\widetilde{N} \rightarrow M_{T_{0}}$ corresponding to $\operatorname{ker} \phi$. Let $f=\phi(\mathrm{f})$ and $h=\phi(\mathrm{h})$, so the deck group of the cover 
is $\phi\left(\Gamma_{T_{0}}\right)=\langle f, h\rangle . \Lambda_{0}$ is the stabilizer of $\mathcal{H}$ in $\operatorname{ker} \phi$, so $\widetilde{S}=\mathcal{H} / \Lambda_{0}$ is a boundary component of $\widetilde{N}$. Since $\left[\Lambda: \Lambda_{0}\right]=5, \widetilde{S}$ is a 5 -fold cover of $\partial_{-} M_{T_{0}}$.

Locate a base point $x \in \partial_{-} M_{T_{0}}$ so that elements of $\Lambda=\pi_{1}\left(\partial_{-} M_{T_{0}}, x\right)$ are represented by loops in $\partial_{-} M_{T_{0}}$, and let $\tilde{x} \in \widetilde{S}$ be in the preimage of $x$. As usual, our choice of the point $\tilde{x}$ gives an action of $\pi_{1}\left(M_{T_{0}}, x\right)$ on $\tilde{N}$. By Lemma 2.4, $f \in \phi(\Lambda)$, so $f^{k} \cdot \tilde{x} \in \widetilde{S}$ for every $k$. Moreover, for each fixed $j,\left(h^{j} f^{k}\right) . \tilde{x}$ and $\left(h^{j} f^{k^{\prime}}\right) \cdot \tilde{x}=\left(h^{j} f^{k}\right) \cdot\left(f^{k^{\prime}-k} \cdot \tilde{x}\right)$ occupy the same component of $\partial \widetilde{N}$ for any $k$ and $k^{\prime}$. This means that $\partial \widetilde{N}$ has eleven components covering $\partial_{-} M_{T_{0}}$. Each component is of the form $h^{j}(\widetilde{S})$ for some $j \in\{0, \ldots, 10\}$ and contains the orbit of $\tilde{x}$ under the left coset $h^{j}\langle f\rangle$. Recall from the proof of 2.4 that $f h=h^{4} f$. Therefore, $\langle f\rangle$ acts on the components of the preimage of $\partial_{-} M_{T_{0}}$ in $\partial \widetilde{N}$. Its three orbits are

$$
\{\widetilde{S}\} \quad\left\{h(\widetilde{S}), h^{4}(\widetilde{S}), h^{5}(\widetilde{S}), h^{9}(\widetilde{S}), h^{3}(\widetilde{S})\right\} \quad\left\{h^{2}(\widetilde{S}), h^{8}(\widetilde{S}), h^{10}(\widetilde{S}), h^{7}(\widetilde{S}), h^{6}(\widetilde{S})\right\} .
$$

Because $C\left(\Omega_{T_{0}}\right)$ is the quotient of $\widetilde{N}$ by the action of $\langle f\rangle$, any component of the latter two orbits projects injectively to $p^{-1}\left(\partial_{-} M_{T_{0}}\right)$. From the proof of Lemma 2.4, we know that $\phi(\mathrm{g})=f h^{-1}=h^{7} f$ and hence $\phi\left(\mathrm{g}^{-1}\right)=h f^{-1}$. Since $h(\widetilde{S})$ and $h^{7}(\widetilde{S})$ lie in different $\langle f\rangle$-orbits the lemma's final claim follows.

Let $\widetilde{M}_{T}$ be the double of $C\left(\Omega_{T_{0}}\right)$ across $p_{T_{0}}^{-1}\left(\left(S^{2} \times\{1\}\right)-T_{0}\right)$ and let $R_{T}: \widetilde{M}_{T} \rightarrow$ $\widetilde{M}_{T}$ be the doubling involution. It is straightforward to show that there is an 11-fold cover $p_{T}: \widetilde{M}_{T} \rightarrow M_{T}$ that restricts on $C\left(\Omega_{T_{0}}\right)$ to $p_{T_{0}}$ such that $p_{T} \circ R_{T}=r_{T} \circ p_{T}$. Let $\partial_{-} \widetilde{M}_{T}=p_{T}^{-1}\left(\partial_{-} M_{T}\right)$.

Corollary 2.7. The mutation of $\partial_{-} M_{T}$ determined by (13)(24) has a hidden extension $\Psi: \widetilde{M}_{T} \rightarrow \widetilde{M}_{T}$ that preserves each component of $p_{T}^{-1}\left(\partial_{-} M_{T}\right)$ and commutes with $R_{T}$.

Proof. Since $\phi\left(\mathrm{m}_{1}\right)$ normalizes $\langle\phi(\mathrm{f})\rangle$ in $S_{12}, \mathrm{~m}_{1}$ normalizes $\Omega_{T_{0}}$. So $\mathrm{m}_{1}$ induces a self-isometry $\Psi_{0}$ of $\mathbb{H}^{3} / \Omega_{T_{0}}$ which, by Fact 1.7 , preserves $M_{T_{0}}$. We claim that $\Psi_{0}$ preserves $p_{T_{0}}^{-1}\left(\left(S^{2} \times\{j\}\right)-T_{0}\right)$ for $j \in\{0,1\}$.

From Lemma 2.3, we know that $\partial C\left(H_{T_{0}}\right)$ has two components. So, under the branched cover $M_{T_{0}} \rightarrow C\left(H_{T_{0}}\right)$, the images of the two components of $\partial M_{T_{0}}$ are distinct. Recall that Proposition 1.1(5) implies $\partial_{-} M_{T_{0}}=\mathcal{H} / \Lambda$. Since each component of $p_{T_{0}}^{-1}\left(\partial_{-} M_{T_{0}}\right)$ is the quotient of a $\Gamma_{T_{0}}$-translate of $\mathcal{H}$ by its stabilizer in $\Omega_{T_{0}}$, this means that the $H_{T_{0}}$ and $\Gamma_{T_{0}}$-orbits of $\mathcal{H}$ are identical. Since $\mathrm{m}_{1} \in \mathrm{PSL}_{2}(\mathbb{Z})<H_{T_{0}}$, the $\Gamma_{T_{0}}$-orbit of $\mathcal{H}$ is perserved by $\mathrm{m}_{1}$. This proves the claim.

Now, using the claim, we obtain an isometry $\Psi$ of $\widetilde{M}_{T}$ that commutes with $R_{T}$ and agrees with $\Psi_{0}$ on $C\left(\Omega_{T_{0}}\right)$. In the last part of the proof of Proposition 6.6 from [3], we show that $\mathrm{m}_{1}$ does not normalize $\Gamma_{T}$. Hence, $\Psi$ is a hidden symmetry of $M_{T}$. Since $\mathrm{m}_{1}$ normalizes $\Lambda, \Psi_{0}$ restricts to a lift of (13)(24) on the component of $p_{T_{0}}^{-1}\left(\partial_{-} M_{T_{0}}\right)$ which is the image of $\mathcal{H}$. Therefore, $\Psi_{0}$ is a hidden extension of this mutation over $C\left(\Omega_{T_{0}}\right)$ and $\Psi$ is a hidden extension over $\widetilde{M}_{T}$.

Corollary 2.8. There is a covering space $p_{S}: \widetilde{M}_{S} \rightarrow M_{S}$ with degree 11 and an isometry $J: \partial \widetilde{M}_{S} \rightarrow \partial_{-} \widetilde{M}_{T}$, which lifts the map $j: \partial M_{S} \rightarrow \partial_{-} M_{T}$, such that $J^{-1} \Psi J$ extends across $\widetilde{M}_{S}$.

Proof. Let $\widetilde{M}_{S}$ be the disjoint union $M_{S} \sqcup \widetilde{N}_{a} \sqcup \widetilde{N}_{b}$, where $\widetilde{N}_{a}$ and $\widetilde{N}_{b}$ are copies of $C\left(\Omega_{0}\right)$. Define $J$ component-wise as follows. 
- Recall the isometric embedding $\iota_{-}^{(0)}: F^{(0)} \rightarrow \partial M_{S}$ and let $\iota_{+}: F^{(0)} \rightarrow$ $\partial \widetilde{M}_{T}$ be the isometric embedding given by Lemma 2.6. Define $\left.J\right|_{\partial M_{S}}$ as $\iota_{+} \circ\left(\iota_{-}^{(0)}\right)^{-1}$.

- Let $\iota_{a}: \mathcal{H} / \Lambda_{0} \rightarrow \partial \widetilde{N}_{a}$ be the isometric embedding guaranteed by Lemma 2.2 and let $\iota_{\mathrm{g}}: \mathcal{H} / \Lambda_{0} \rightarrow \partial \widetilde{M}_{T_{0}}$ be the composition of the isometric embedding $\mathrm{g}(\mathcal{H}) / \mathrm{g} \Lambda_{0} \mathrm{~g}^{-1} \rightarrow \partial_{-} \widetilde{M}_{T_{0}}$ given by Lemma 2.6 with the natural isometry $\mathcal{H} / \Lambda_{0} \rightarrow \mathrm{g}(\mathcal{H}) / \mathrm{g} \Lambda_{0} \mathrm{~g}^{-1}$. Define $\left.J\right|_{\partial \widetilde{N}_{a}}$ as $\iota_{\mathrm{g}} \circ \iota_{a}^{-1}$.

- Define $\left.J\right|_{\partial \widetilde{N}_{b}}$ as $\iota_{\mathrm{g}^{-1}} \circ \iota_{b}^{-1}$ in analogy with the case above.

To see that $J$ lifts $j$, notice first that Proposition 1.2 implies that $j=\iota_{+}^{(0)}\left(\iota_{-}^{(0)}\right)^{-1}$. Now, for $x \in \mathbb{H}^{3}$, the covering map $\mathbb{H}^{3} / \Omega_{0} \rightarrow \mathbb{H}^{3} / \Delta_{0}$ sends $\Omega_{0}(x)$ to $\Gamma_{0}(x)$ and $\mathcal{H} / \Lambda_{0} \rightarrow F^{(0)}$ sends $\Lambda_{0}(x)$ to $\Lambda(x)$. So $\iota_{a}$ and $\iota_{b}$ lift $\iota_{0}$. Similarly, $\iota_{\mathrm{g}}$ and $\iota_{\mathrm{g}^{-1}}$ lift $\iota_{+}^{(0)}$, since $\iota_{+}^{(0)}$ factors as the natural composition $F^{(0)} \rightarrow \times(\mathcal{H}) / \times \Lambda \mathrm{x}^{-1} \rightarrow \partial_{-} M_{T}$ whenever $x \in \Gamma_{T}$.

Lemma 5.8 of [3] implies that $\mathrm{m}_{1}$ normalizes $\Delta_{0}$. So, the restriction of $J^{-1} \Psi J$ to $\partial M_{S}$ extends over $M_{S}$. A calculation shows that $\mathrm{gm}_{1} \mathrm{~g}^{-1}=\left(\begin{array}{c}1-1 \\ 2-1\end{array}\right)$, so $\mathrm{gm}_{1} \mathrm{~g}^{-1}$ preserves $\mathcal{H}$ and $\mathrm{m}_{1}$ preserves $\mathrm{g}^{-1}(\mathcal{H})$. For $x \in \mathcal{H}$, the map $\left.J^{-1} \Psi J\right|_{\partial \widetilde{N}_{a}}$ takes $\Omega_{0}(x)$ to $\Omega_{0}\left(\mathrm{~g}^{-1} \mathrm{~m}_{1} \mathrm{~g}(x)\right)$. This map extends over $\tilde{N}_{a}$ since $\mathrm{g}^{-1} \mathrm{~m}_{1} \mathrm{~g}(x) \in H_{0}$ and, by Lemma $2.2, \Omega_{0}$ is normal in $H_{0}$.

Since $\Psi$ takes $\partial_{-} \widetilde{M}_{T}$ to itself and preserves the components covered by $\mathcal{H}$ and $\mathrm{g}^{-1}(\mathcal{H})$, it also preserves the component covered by $\mathrm{g}(\mathcal{H})$. For $x \in \mathcal{H}$, the map $\left.J^{-1} \Psi J\right|_{\partial \widetilde{N}_{b}}$ takes $\Omega_{0}(x)$ to $\Omega_{0}\left(\mathrm{gm}_{1} \mathrm{~g}^{-1}(x)\right)$. As before, this map extends over $\widetilde{N}_{b}$ because $\Omega_{0}$ is normal in $H_{0}$.

Theorem 2.9. For each $n \in \mathbb{N}$ there is an 11-sheeted cover $N_{n} \rightarrow B^{3}-T_{n}$ and a hidden extension $\Psi: N_{n} \rightarrow N_{n}$ of the mutation (13)(24) acting on $S^{(n)}-T_{n}$. Moreover, for each $m \in \mathbb{N}, \Psi$ extends to a hidden symmetry of an 11-sheeted cover of $S^{3}-L_{m+n}$ that contains $N_{n}$.

Proof. Let $\partial_{+} \widetilde{M}_{T}^{(j)}=\partial \widetilde{M}_{T}^{(j)}-\partial_{-} \widetilde{M}_{T}^{(j)}$ and compose $R_{T}: \partial_{+} \widetilde{M}_{T} \rightarrow \partial_{-} \widetilde{M}_{T}$ with marking maps to obtain isomorphisms $R_{T}: \partial_{+} \widetilde{M}_{T}^{(j)} \rightarrow \partial_{-} \widetilde{M}_{T}^{(j+1)}$. Define $N_{n}$ to be the adjunction space

$$
\widetilde{M}_{S} \cup_{J} \widetilde{M}_{T}^{(1)} \cup_{R_{T}} \cdots \cup_{R_{T}} \widetilde{M}_{T}^{(n)} .
$$

Because $J$ lifts $j$ and $R_{T}$ lifts $r_{T}$, the covering maps $p_{S}$ and $p_{T}$ determine covering spaces $p_{n}: N_{n} \rightarrow B^{3}-T_{n}$, which restrict to $p_{S}$ and $p_{T}$ on the factors of the adjunction space.

The hidden extension $\Psi$ is given on each of the $\widetilde{M}_{T}^{(i)}$ by the eponymous symmetry from Corollary 2.7 and on $\widetilde{M}_{S}$ by the extension of $J^{-1} \Psi J$ described in Corollary 2.8. Corollary 2.7 implies that $\Psi: N_{n} \rightarrow N_{n}$ does not descend to $B^{3}-T_{n}$.

Let $\bar{p}_{n}: \bar{N}_{m} \rightarrow \overline{B^{3}-T_{m}}$ be the mirror image of the cover $p_{n}$ and $\bar{\Psi}: \bar{N}_{m} \rightarrow$ $\bar{N}_{m}$ the mirror image of $\Psi$. Using the mirror map $\partial N_{n} \rightarrow \partial N_{m}$ to glue, we form an adjunction space $\widetilde{M}_{m+n}=N_{n} \cup \bar{N}_{m}$. The covering maps $p_{n}$ and $\bar{p}_{n}$ determine a covering space $\widetilde{M}_{m+n} \rightarrow S^{3}-L_{m+n}$ and $\Psi$ and $\bar{\Psi}$ determine an isometry $\widetilde{M}_{m+n} \rightarrow \widetilde{M}_{m+n}$. As defined, the covering space and isometry restrict to $p_{n}$ and $\Psi$ on $N_{n} \subset \widetilde{M}_{m+n}$. 
Remark 2.10. The referee has asked whether the covers of $B^{3}-T_{n}$ and $S^{3}-$ $L_{m+n}$ described in Theorem 2.9 have minimal degree among those admitting hidden extensions of (13)(24). We suspect this is so but cannot quite prove it. Below we prove a related but weaker assertion. Suppose $\Phi: M_{1} \rightarrow M_{2}$ is a hidden symmetry of $M_{T_{0}}$ that restricts to a lift of $(13)(24)$ on a component $S_{1}$ of $p_{1}^{-1}\left(\partial_{-} M_{T}\right)$, where $p_{1}: M_{1} \rightarrow M_{T_{0}}$ and $p_{2}: M_{2} \rightarrow M_{T_{0}}$ are finite-degree connected covers of $M_{T_{0}}$. If $\Phi$ is induced by some $\mathrm{n} \in H_{T_{0}}$ from Lemma 2.3 then the $p_{i}$ have degree at least 11 .

Let $\Gamma_{1}$ and $\Gamma_{2}$ be the finite-index subgroups of $\Gamma_{T_{0}}$ respectively corresponding to the $M_{i}$. There exist finite-index subgroups $\Lambda_{i}$ of $\Lambda$, and $g_{i} \in \Gamma_{T_{0}}-\Gamma_{i}$ so that $S_{1}$ is represented in $\Gamma_{1}$ by $g_{1} \Lambda_{1} g_{1}^{-1}$ and $S_{2}=\Phi\left(S_{1}\right)$ by $g_{2} \Lambda_{2} g_{2}^{-1}$. For each $i$, the restriction of $p_{i}$ to $S_{i}$ has $p_{i *}\left(g_{i} \lambda g_{i}^{-1}\right)=\lambda$ for $\lambda \in \Lambda_{i}$. That $\Phi: S_{1} \rightarrow S_{2}$ lifts (13)(24) translates at the level of induced maps to

$$
\mathrm{m}_{1} \lambda \mathrm{m}_{1}^{-1}=g_{2}^{-1} \Phi_{*}\left(g_{1} \lambda g_{1}^{-1}\right) g_{2}=g_{2}^{-1} \mathrm{n} g_{1} \lambda g_{1}^{-1} \mathrm{n}^{-1} g_{2}
$$

for each $\lambda \in \Lambda_{1}$, since $\mathrm{m}_{1}: \Lambda \rightarrow \Lambda$ is the induced map of (13)(24). But the centralizer of $\Lambda_{1}$ in $\mathrm{PSL}_{2}(\mathbb{C})$ is trivial, so we have $\mathrm{n}=g_{2} \mathrm{~m}_{1} g_{1}^{-1}$. Lemma 2.4(3) now implies that $\Gamma_{1}$ has index at least 11 in $\Gamma_{T_{0}}$.

Acknowledgement. We are grateful to the referee for asking interesting questions, and for suggesting changes that have improved the quality of this paper.

\section{REFERENCES}

[1] I. R. Aitchison and J. H. Rubinstein. Combinatorial cubings, cusps, and the dodecahedral knots. In Topology '90 (Columbus, OH, 1990), volume 1 of Ohio State Univ. Math. Res. Inst. Publ., pages 17-26. de Gruyter, Berlin, 1992.

[2] Michel Boileau, Steven Boyer, Radu Cebanu, and Genevieve S. Walsh. Knot commensurability and the Berge conjecture. Geom. Topol., 16(2):625-664, 2012.

[3] Eric Chesebro and Jason DeBlois. Algebraic invariants, mutation, and commensurability of link complements. Pacific J. Math., 267(2):341-398, 2014.

[4] David Coulson, Oliver A. Goodman, Craig D. Hodgson, and Walter D. Neumann. Computing arithmetic invariants of 3-manifolds. Experiment. Math., 9(1):127-152, 2000.

[5] Marc Culler, Nathan M. Dunfield, Matthias Goerner, and Jeffrey R. Weeks. SnapPy, a computer program for studying the geometry and topology of 3-manifolds. Available at http://snappy . computop. org.

[6] Nathan Dunfield. Private communication.

[7] Neil Hoffman. On knot complements that decompose into regular ideal dodecahedra. Preprint. arXiv:1209.1004, September 2012. To appear, Geom. Dedicata.

[8] Melissa L. Macasieb and Thomas W. Mattman. Commensurability classes of $(-2,3, n)$ pretzel knot complements. Algebr. Geom. Topol., 8(3):1833-1853, 2008.

[9] Walter D. Neumann and Alan W. Reid. Arithmetic of hyperbolic manifolds. In Topology '90 (Columbus, OH, 1990), volume 1 of Ohio State Univ. Math. Res. Inst. Publ., pages 273-310. de Gruyter, Berlin, 1992.

[10] Alan W. Reid. Arithmeticity of knot complements. J. London Math. Soc. (2), 43(1):171-184, 1991.

[11] Alan W. Reid and Genevieve S. Walsh. Commensurability classes of 2-bridge knot complements. Algebr. Geom. Topol., 8(2):1031-1057, 2008.

[12] W. P. Thurston. The geometry and topology of 3-manifolds. mimeographed lecture notes, 1979.

[13] Robert J. Zimmer. Ergodic theory and semisimple groups, volume 81 of Monographs in Mathematics. Birkhäuser Verlag, Basel, 1984. 
Department of Mathematical Sciences, University of Montana

E-mail address: Eric.Chesebro@mso.umt.edu

Department of Mathematics, University of Pittsburgh

E-mail address: jdeblois@pitt.edu 